\title{
In-situ temperature calibration procedure for temperature and strain fibre Bragg grating sensors for monitoring pre-stressing strands
}

\author{
I. Mckeeman*a ${ }^{\mathrm{a}}$, G. Fusiek ${ }^{\mathrm{a}}$, M.Perry ${ }^{\mathrm{a}}$, P. Niewczas ${ }^{\mathrm{a}}$, M. Johnston ${ }^{\mathrm{b}}$ \\ ${ }^{a}$ Department of Engineering, University of Strathclyde, Glasgow, United Kingdom \\ ${ }^{\mathrm{b}}$ Civil Design Group, EDF Energy, Nuclear Generation, East Kilbride, United Kingdom \\ *iain.mckeeman@strath.ac.uk
}

\begin{abstract}
In this work, we demonstrate active and passive methods for in-situ temperature calibration of fibre Bragg grating strain and temperature sensors. The method is suitable for characterizing sensors which are already attached to the steel reinforcements of civil structures. The proposed method, which involves the use of active induction heating or passive room temperature fluctuations, can be implemented using portable equipment, is time efficient, and can be used to calibrate attached sensors on-site, rather than in lab conditions. Preliminary results of the induction heating calibration show good agreement with pre-calibrated temperature sensors. In-situ calibration of fibre strain sensors, attached to a prestressing strand is also successfully carried out.
\end{abstract}

Keywords: prestress monitoring, fibre Bragg gratings, structural health monitoring, temperature calibration

\section{INTRODUCTION AND MOTIVATION}

Optical sensors are becoming increasingly common in structural health monitoring (SHM) applications [1], [2]. Fibre Bragg gratings (FBGs) can provide quasi-distributed monitoring of strain and temperature within civil structures. As with all strain sensors in SHM, strain FBG sensors require temperature compensation in order to properly describe the mechanical strain of the substrate or the structure it is embedded into or bonded to, independently of thermal expansion [3].

For most temperature compensation methods to be valid, the thermal response of a strain FBG sensor must be accurately known. Controlled temperature characterisation of individual sensors is challenging in SHM applications as the structures are large. As such, sensors may be calibrated in an environmental chamber before attachment to substrates, but this is inconvenient and inaccurate, as it requires the thermal expansion of the substrate and also the strain transfer between the substrate and the sensor to be known. Furthermore, in field applications, it is often assumed that all sensors have the same response, but verification of each sensor's response can provide a much higher level of measurement accuracy.

Continuing the research outlined in [4], we have developed a portable, in-situ method for obtaining the temperature response coefficients for FBG sensors which are attached to prestressing strands. Two methods of temperature calibration are outlined. The first is an active method, in which the FBGs are locally heated using an induction heater. The second method monitors the wavelength shifts passively during natural, daily temperature fluctuations in room temperature. By comparing these two measurement techniques, the first with its large range and larger temperature instabilities, the latter with its smaller range and smaller temperature instabilities, a reliable temperature coefficient for FBG sensors can be derived. This work reports on preliminary results of the calibration procedure by comparing precalibrated FBG sensors with those calibrated using the in-situ method.

\section{THEORY}

The wavelength response of an FBG under strain, $\varepsilon$ and changing temperature, $\Delta T$ is given by:

$$
\Delta \lambda_{B} / \lambda_{B}=K_{\varepsilon} \varepsilon+K_{T} \Delta T
$$


where $\mathrm{K}_{\mathrm{g}}$ and $\mathrm{K}_{\mathrm{T}}$ are the FBG's strain and temperature coefficients respectively. It is common practice to decouple the strain and temperature response of an FBG by comparing its wavelength shifts with a second, local temperature reference FBG which is isolated from strain. For a high accuracy measurements, it is crucial to characterize the responses of both sensors after they have been packaged and attached to a substrate, as manufacturing processes may lead to changes in strain transfer and thermal expansion, and hence changes in $\mathrm{K}_{\mathrm{z}}$ and $\mathrm{K}_{\mathrm{T}}$. By characterising the temperature response of sensors in-situ, temperature coefficients can be conveniently and accurately measured.

\section{SENSORS AND CALIBRATION PROCEDURE}

\section{Sensor fabrication and attachment}

The sensors in this demonstration were fabricated from type-I FBGs, brazed into a robust metal package using an induction coil as discussed in previous work [5]. The temperature sensors were attached to prestressing strands at one end using silicone, to ensure thermal contact without strain transfer. The strain FBG sensors were spot welded to the strand via metal shims (Fig 1).

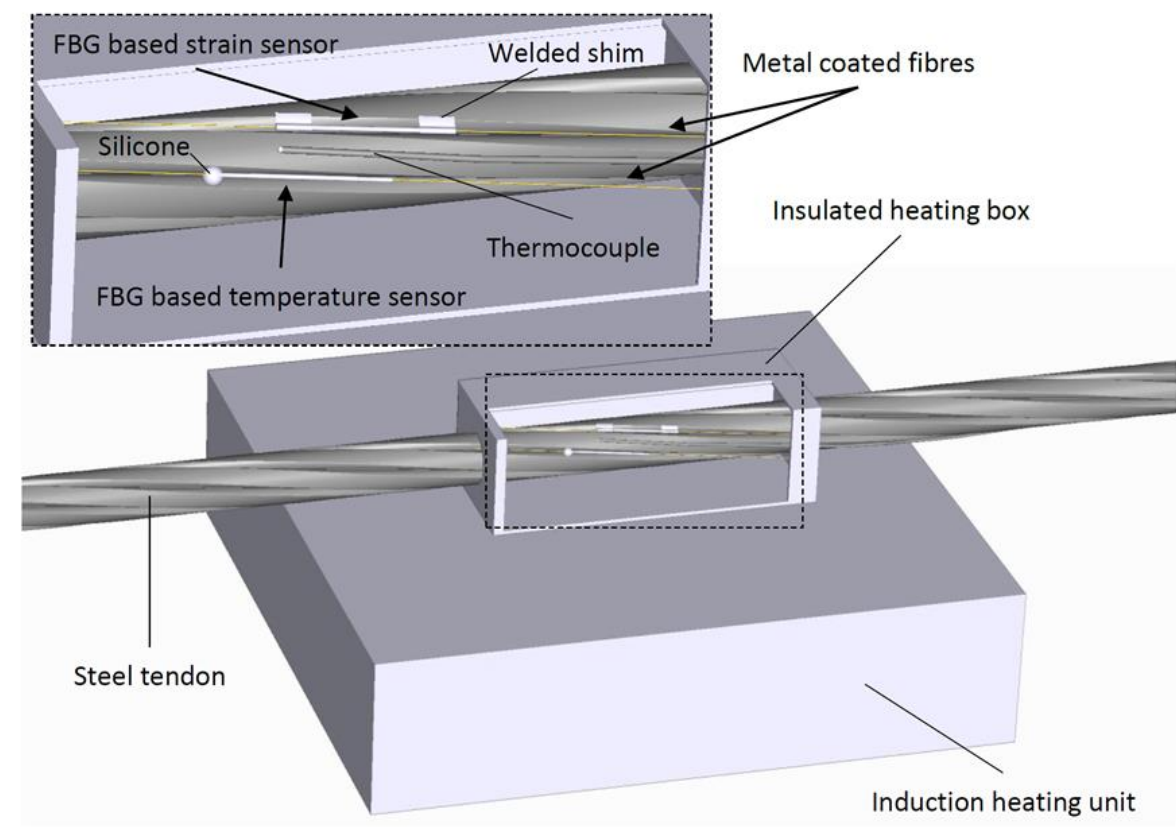

Figure 1. Induction heating set-up, an insulated metal box surrounds the section of strand where the sensors are attached. The thermocouple was situated in between the two sensors and in contact with the strand.

\section{Sensor calibration procedure}

Temperature FBG sensors were calibrated in an environmental chamber. They were subject to four heating cycles from $10-50^{\circ} \mathrm{C}$, the temperature was stepped in $10^{\circ} \mathrm{C}$ intervals and held at each point for two hours to allow the temperature to stabilise. These sensors were attached to the pre-stressing strand next to the strain sensors (Figure 1). The pre-calibration of the temperature sensors allowed the calibration techniques below to be validated.

Active heating method - In the active method, an induction heater is used to heat a section of steel strand at the location of each strain sensor. To allow higher temperatures to be realised, strand sections are also heated indirectly by enveloping them in an insulated steel box (Figure 1). The box acts as a heat susceptor and also reduces convective heat loss. The strand is heated to approximately $60^{\circ} \mathrm{C}$ and then left to cool. The wavelength response of the strain sensor is compared to an in-situ temperature FBG (pre-calibrated) and a J-type thermocouple $\left( \pm 1^{\circ} \mathrm{C}\right.$ accuracy), placed in thermal contact with the strand using a thermally conductive gel.

Passive heating method - Fluctuations in room temperature can also be monitored over several days along with FBG wavelength shifts to accomplish a passive temperature calibration technique. The change in room temperature is 
relatively slow so there is less likelihood of variations in temperature between the sensors and the thermocouple. In this work, the natural range of temperature variation was, at most, $7^{\circ} \mathrm{C}$ each day.

\section{RESULTS}

\section{Pre calibrated temperature sensor results}

The rapid and non-uniform temperature increases during induction heating led to temperature instabilities and variations between the sensors and the strand. As such, only cooling of the strand, exhibiting much slower temperature variation, was used to characterize temperature coefficients. In each case, it took approximately 3.5 hours for strand sections to decay from $60^{\circ} \mathrm{C}$ to room temperature.

Figure 2 shows the wavelength response of the pre-calibrated temperature sensor compared to temperature measured by the thermocouple during cooling from induction heating. The temperature coefficient matches the previous calibration $\left(15.9 \mathrm{pm} /{ }^{\circ} \mathrm{C}\right)$ within the error estimate. The thermocouple data plotted shows a relatively high noise level; comparing the variance between the thermocouple and the FBG data, $x$ vs $y$, indicates that a more accurate temperature reference will reduce the error and increase the accuracy of the calibration substantially.
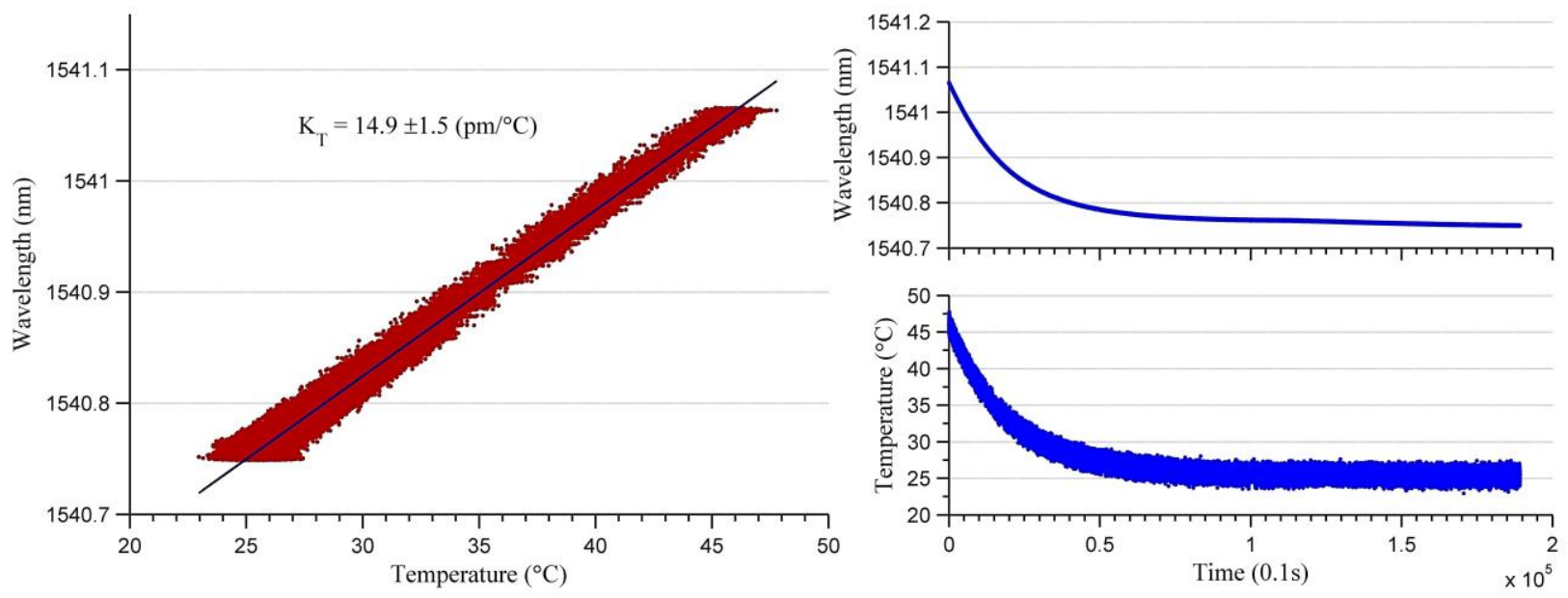

Figure 2. Temperature response of a temperature FBG sensor during cooling after induction heating (left). The cooling profiles of the FBG (top) and thermocouple (bottom) can be seen on the right.
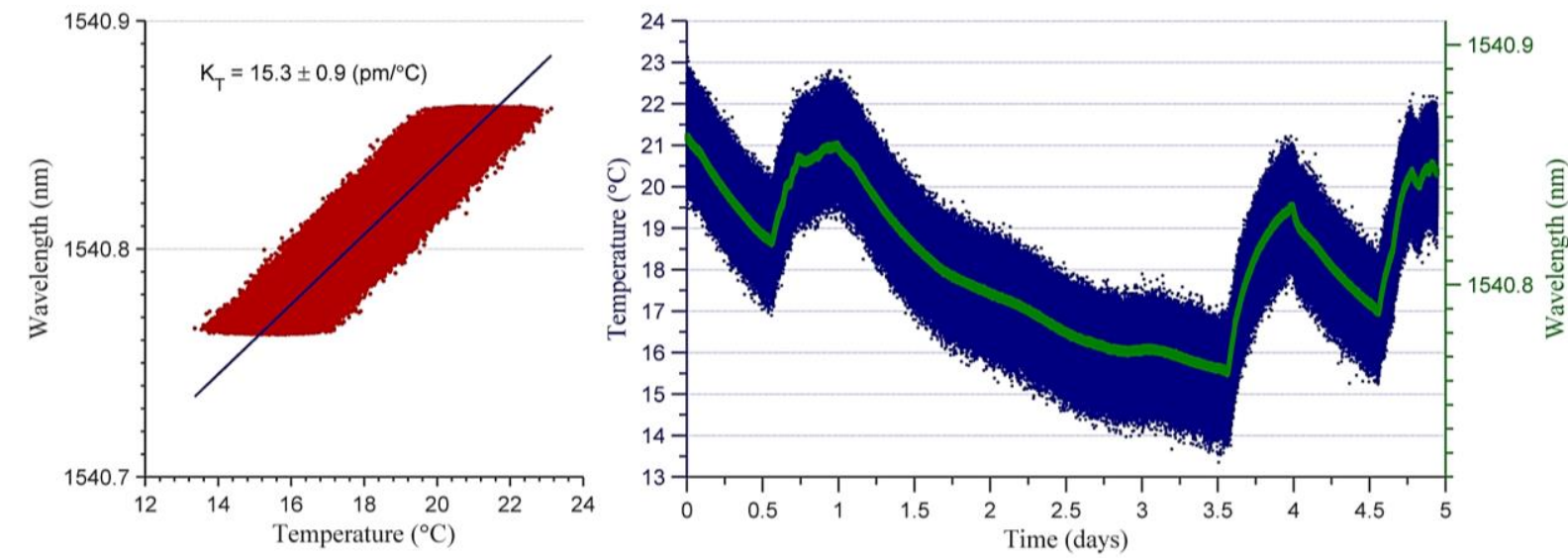

Figure 3. Temperature response of the same temperature FBG sensor during room temperature fluctuations (left). The FBG sensor is able to measure smaller deviations in the temperature compared to the thermocouple (right).

The results from room temperature fluctuations can be seen in Figure 3. The FBG temperature sensor is able to repeat the thermocouple trend with additional features seen due to the high resolution of the sensor. A calibration coefficient of 
$15.3 \mathrm{pm} /{ }^{\circ} \mathrm{C}$ was obtained from these measurements; this is comparable to the coefficient obtained from induction heating. These results show the temperature characterization method proposed is a viable solution to this practical problem.

\section{Strain sensor calibration results}

Finally, the procedure was repeated for a strain $\mathrm{FBG}$ sensor, yielding values of $18.1 \pm 1.8 \mathrm{pm} /{ }^{\circ} \mathrm{C}$ and $17.1 \pm 1.0 \mathrm{pm} /{ }^{\circ} \mathrm{C}$ (induction heating and room temperature variation respectively) for its temperature coefficients. This is larger than the temperature FBG sensor due to the thermal expansion of steel that is also present in its temperature dependence. Temperature variations while measuring strain can now be compensated for, allowing the mechanical strain of the steel strands to be determined.
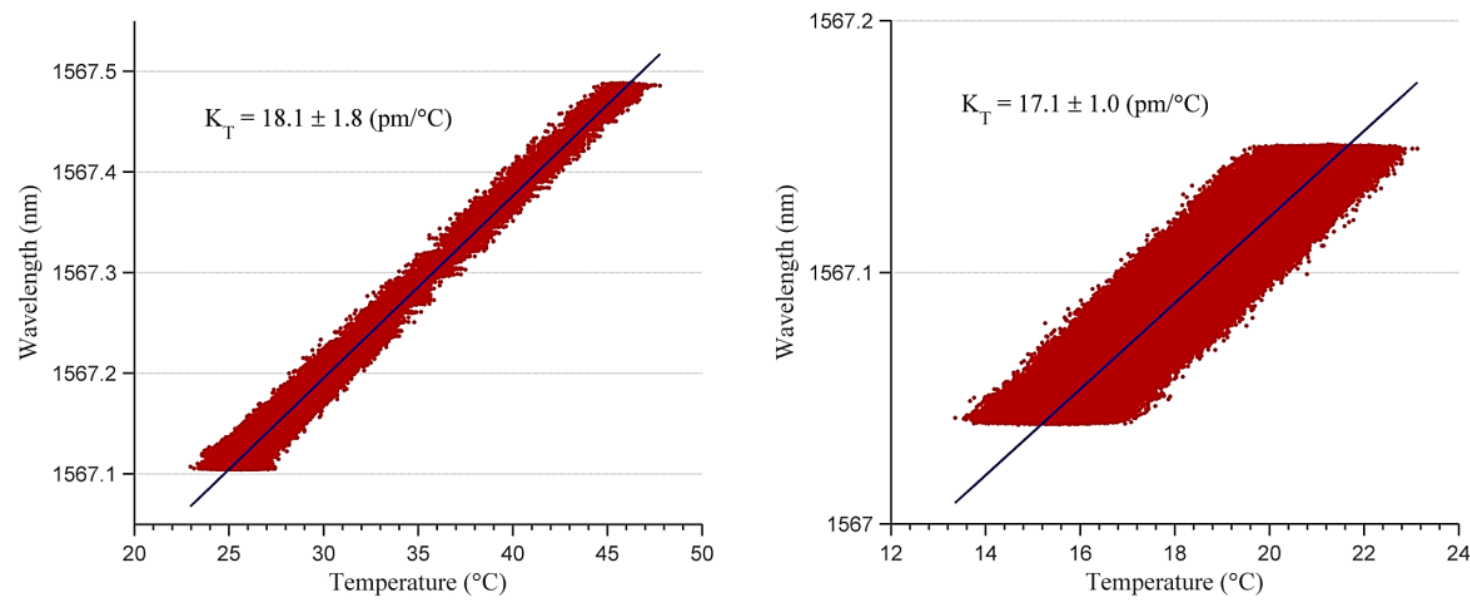

Figure 4. Temperature calibration of a strain FBG sensor using the induction heating method (left). Comparison to room temperature fluctuations (right)

\section{CONCLUSION}

We have outlined a practical solution for calibrating in-situ strain sensors, mounted on steel strands for prestress monitoring. Tests with a pre-calibrated temperature fibre Bragg grating sensor show that active method of induction heating is capable of calibrating temperature sensors to within $1.5 \mathrm{pm} /{ }^{\circ} \mathrm{C}$ error. This error may be reduced by using more accurate temperature references. Future work will concentrate on testing pre-calibrated strain FBG sensors to further validate this temperature calibration technique.

\section{ACKNOWLEDGEMENTS}

This work was supported by the Engineering and Physical Sciences Research Council (EPSRC) and EDF Energy Nuclear Generation, Ltd.

\section{REFERENCES}

[1] H.-N. Li, D.-S. Li, and G.-B. Song, "Recent applications of fiber optic sensors to health monitoring in civil engineering,” Eng. Struct., vol. 26, no. 11, pp. 1647-1657, Sep. 2004.

[2] J. M. Lopez-Higuera, L. Rodriguez Cobo, A. Quintela Incera, and A. Cobo, "Fiber Optic Sensors in Structural Health Monitoring," J. Light. Technol., vol. 29, no. 4, pp. 587-608, Feb. 2011.

[3] M. Majumder, T. K. Gangopadhyay, A. K. Chakraborty, K. Dasgupta, and D. K. Bhattacharya, "Fibre Bragg gratings in structural health monitoring-Present status and applications," Sens. Actuators Phys., vol. 147, no. 1, pp. 150-164, Sep. 2008.

[4] M. Perry, Z. Yan, Z. Sun, L. Zhang, P. Niewczas, and M. Johnston, "High stress monitoring of prestressing tendons in nuclear concrete vessels using fibre-optic sensors," Nucl. Eng. Des., vol. 268, pp. 35-40, Mar. 2014.

[5] M. Perry, P. Niewczas, M. Johnston, K. Cook, and J. Canning, "Induction Brazing of Type-I Fiber Bragg Gratings Into Kovar Ferrules Exploiting Curie Transition,” IEEE Sens. J., vol. 13, no. 2, pp. 816-823, Feb. 2013. 